\title{
Intermittent versus Daily Eltrombopag Dosage Protocols for the Treatment of Primary Immune Thrombocytopenia: Real-Life Experience
}

This article was published in the following Dove Press journal: Journal of Blood Medicine

\author{
Tamer A Elbedewy $\mathbb{D}^{\prime}$ \\ Mohamed A Elsebaey' \\ Reem A Elkholy ${ }^{2}$ \\ Dina M Tahoon ${ }^{2}$ \\ Samah A Elshweikh' \\ 'Internal Medicine Department, Faculty \\ of Medicine, Tanta University, Tanta, \\ Egypt; ${ }^{2}$ Medical Pharmacology \\ Department, Faculty of Medicine, Tanta \\ University, Tanta, Egypt
}

Introduction/Aim: Eltrombopag is recommended for the treatment of refractory immune thrombocytopenia (ITP). Based on its half-life, it may be practical to use an intermittent dosage. Our aim was to compare the effectiveness and safety of intermittent vs daily eltrombopag dosage protocols for the treatment of primary ITP refractory to prior therapies. Patients and Methods: This was a retrospective study, and 34 adult primary ITP patients refractory to prior therapies were included in our analysis. Eltrombopag was used in this study. The patients were divided into daily eltrombopag dosage and intermittent eltrombopag dosage groups. Eltrombopag effectiveness was assessed regarding platelet count and bleeding resolution. Safety was assessed via adverse events reporting.

Results: In the daily eltrombopag dosage group, overall response (OR), complete response (CR), partial response (PR), and relapse rates were 69.23\%, 53.85\%, 15.38\%, and $30.77 \%$, respectively. In the intermittent eltrombopag dosage group, OR, CR, PR, and relapse rates were $68.75 \%, 50 \%, 18.75 \%$, and $31.25 \%$, respectively. Comparison between daily and intermittent eltrombopag dosage groups as regards CR, PR, relapse, relapse-free survival and adverse events showed insignificant differences.

Conclusion: Intermittent eltrombopag dosage is safe and effective in patients with ITP refractory to prior therapies and comparable to the daily eltrombopag dosage.

Keywords: immune thrombocytopenia, eltrombopag, daily dosage, intermittent dosage, effectiveness, safety

\section{Introduction}

Immune thrombocytopenia (ITP) is an autoimmune disorder characterized by low number of circulating platelets owing to their destruction in the spleen as well as the inability of bone marrow megakaryocytes to restore normal platelet counts. ${ }^{1,2}$

Corticosteroids have been considered the standard first-line of treatment for patients with ITP. ${ }^{3}$ However, failure of response to these agents is common, and treatment of these patients with refractory ITP is unsatisfactory and constitutes a matter of controversy. ${ }^{4,5}$ Interestingly, daily clinical practice demonstrates that, in spite of the current guidelines which considered splenectomy as a suitable secondchoice therapy for ITP, this is being substituted with thrombopoietin receptor (TPOR) agonist therapy. 6,7

For many years, the use of one of these agents, namely eltrombopag, has been recommended for treatment of ITP patients refractory to first-line therapy or splenectomy. ${ }^{8}$ Eltrombopag interacts with TPO-R. As a consequence, it enhances
Correspondence: Tamer A Elbedewy Department of Internal Medicine, Tanta University, Tanta, Egypt

Tel + 020-01094680720

Email tamer.elbedawi@med.tanta.edu.eg 
megakaryocytes proliferation and differentiation to induce recovery of platelet counts. ${ }^{9}$ Based on its half-life in ITP patients (26-35 hours), ${ }^{10,11}$ it may be practical to use intermittent eltrombopag dosage as an alternative to its standard daily dosage for treatment of those patients.

It is worth noting that this study were performed to evaluate the intermittent eltrombopag dosage protocol as an alternative to its daily dosage for treatment of patients with refractory ITP, paying attention to the effectiveness and safety in real-life experience.

The aim of the present study was to compare the effectiveness and safety of intermittent versus daily eltrombopag dosage protocols for the treatment of primary persistent and chronic immune thrombocytopenia refractory to prior therapies.

\section{Patients and Methods Patients and Eligibility Criteria}

This retrospective study was conducted at the Hematology Unit, Internal Medicine Department, Tanta University and Tanta Insurance Hospital in the period between August 2017 and February 2020. Thirty-four consecutive patients with ITP were included in our analysis. Eligible patients were aged $\geq 18$ years, suffered from primary ITP for $>3$ months (persistent or chronic), and were refractory to prior ITP therapies (platelet count $<30 \times 10^{9} / \mathrm{L}$ ). The diagnosis of primary ITP and the definitions of persistent and chronic ITP were set according to the recent guidelines. $^{12,13}$

Patients with newly diagnosed or secondary immune thrombocytopenia were excluded. Prior splenectomy was performed and prior therapy with immunosuppressive drugs, and/or rituximab were administered at least a 6-month interval before initiating eltrombopag. Patients with incomplete medical records or a different eltrombopag protocol from our study protocol were also excluded.

\section{Treatment Protocol}

Eltrombopag (Revolade ${ }^{\circledR}$; Novartis Pharmaceuticals UK Ltd) was used in this study. The initial daily eltrombopag dose was $50 \mathrm{mg}$ for 2 weeks. This daily dose was increased by $25 \mathrm{mg}$ for patients with platelet count $<50 \times 10^{9} / \mathrm{L}$ (maximum daily dose was $75 \mathrm{mg}$ for another 2 weeks). At this point, eltrombopag therapy was discontinued in patients who still had platelet count $<50 \times 10^{9} / \mathrm{L}$ and excluded from this study. After excluding those patients, the remaining patients were divided into two groups. Daily eltrombopag dosage group: patients with platelet count ranging from $50 \times 10^{9} / \mathrm{L}$ to $199 \times 10^{9} / \mathrm{L}$. Intermittent eltrombopag dosage: patient with platelet count $\geq 200 \times 10^{9} / \mathrm{L}$. Intermittent eltrombopag dosage group patients who failed to maintain a platelet count $\geq 50 \times 10^{9} / \mathrm{L}$ on 3 days/week dosage were shifted to the daily eltrombopag dosage group (Figure 1). ${ }^{10}$

\section{Follow-Up}

Platelet count and clinical assessment were done weekly during the first 4 weeks of therapy or after any dose change and then every 4 weeks.

Rescue therapies such as steroids, romiplostim, or platelet transfusions were required for relapsed ITP patients.

\section{Effectiveness Analysis}

Eltrombopag effectiveness was assessed regarding platelet count and bleeding resolution. Complete response (CR): platelet count $>100 \times 10^{9} / \mathrm{L}$ without clinically relevant bleeding. Partial response (PR): platelet count 30$100 \times 10^{9} / \mathrm{L}$ with at least doubling pretreatment platelet count as well as no clinically relevant bleeding. Overall response (OR): $\mathrm{CR}+\mathrm{PR}$. No response (NR): platelet count $<30 \times 10^{9} / \mathrm{L}$, less than a doubling of the pre-treatment platelet count or with clinically relevant bleeding with the highest approved dose (75 mg per day) of eltrombopag for 4 consecutive weeks. Relapse: platelet count $<30 \times 10^{9} / \mathrm{L}$, or clinically relevant bleeding after CR or PR. ${ }^{14}$

Bleeding severity associated with ITP was assessed using the World Health Organization (WHO) bleeding scale (Grade 0: no bleeding, Grade 1: petechiae, Grade 2: mild bleeding, Grade 3: gross bleeding, Grade 4: debilitating bleeding). ${ }^{15}$

\section{Safety Analysis}

Safety was assessed via adverse events recording especially cataract, hepatobiliary laboratory abnormalities, thrombosis, serious infection, bone marrow fibrosis, and death. Adverse events were assessed according to the National Cancer Institute's Common Terminology Criteria for Adverse Events version 5. ${ }^{16}$

\section{Data Collection}

Patient data which were collected from the patient records included: age, sex, ITP diagnosis date, previous ITP therapies, history of splenectomy, bleeding grades before and during eltrombopag therapy, platelet counts before and through eltrombopag therapy, minimum and maximum 


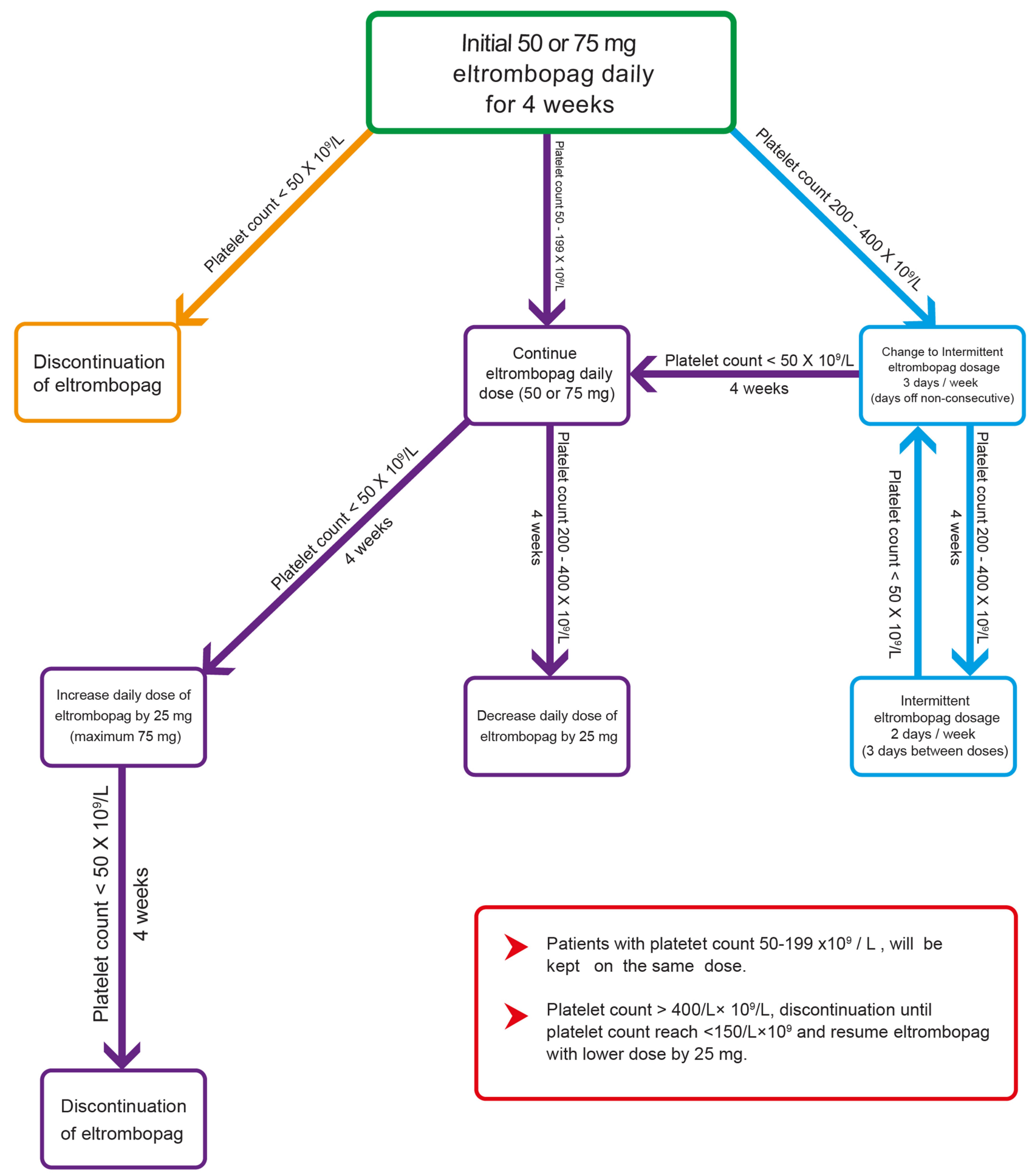

Figure I Eltrombopag dosage protocol.

platelet count during therapy, eltrombopag doses, minimum and maximum doses of eltrombopag, duration of treatment, treatment response rates, duration of treatment response rates, the cause of discontinuation, adverse events of eltrombopag, and rescue therapies.

\section{Clinical Outcome}

The primary outcome was relapse-free survival (RFS) estimation which was calculated as the time from date of the treatment initiation to the date of relapse, death due to any cause, or the end date of the study. 


\section{Statistical Analysis}

The statistical analysis was performed using statistical package for social sciences (SPSS) version 22.0 for Windows (SPSS Inc., Chicago, IL). Analysis was primarily descriptive. Data were expressed as absolute number and percentage for categorical variables, mean \pm standard deviation for continuous parametric variables or median for continuous non-parametric variables. Fisher exact test and Chi-square tests were used for comparison between categorical variables. Comparison between two groups was made by using unpaired $t$-test or Mann-Whitney test for continuous parametric and non-parametric variables, respectively. RFS was done by Kaplan-Meier survival curve using log rank test. A $P$-value $<0.05$ was considered statistically significant.

\section{Results}

\section{Patient Characteristics}

Our study included 34 patients with chronic or persistent ITP, refractory to the previous therapies. The mean age was $35.29 \pm 9.23(19-51)$ years. There were 23 females (67.65\%) with the female:male ratio approximately $2: 1$. The mean ITP duration was $21.88 \pm 9.75$ (7-42) months. The mean initial platelet count at diagnosis was 19.44 $\pm 3.39(13-27) \times 10^{9} / \mathrm{L}$. All of the patients received steroids and platelet transfusion as previous therapy, $28(82.35 \%)$ had previously been treated with other immunosuppressive drugs, 19 (55.88\%) had been treated with vincristine, eight (23.53\%) had received danazol, four (11.76\%) had received prior thrombopoietin mimetics, and four $(11.76 \%)$ had received rituximab. Nine $(26.47 \%)$ had a history of splenectomy. There were $11(32.35 \%), 16$ (47.06\%), and seven $(20.59 \%)$ patients with WHO grade 1,2 , and 3 of bleeding before eltrombopag therapy, respectively.

All the patients received the daily dose of eltrombopag for 4 weeks. In five patients (14.71\%) eltrombopag therapy was discontinued due to platelet count $<50 \times 10^{9} / \mathrm{L}$ and they were excluded from this study. The remaining 29 patients (85.29\%) had a platelet count $\geq 50 \times 10^{9} / \mathrm{L}$ and were divided into two groups - the daily and intermittent eltrombopag dosage groups (Figure 2).

\section{Platelet Count}

Comparison between the daily and intermittent eltrombopag dosage groups as regard the platelet count (initial at diagnosis, maximum during eltrombopag treatment, and minimum during eltrombopag treatment) showed insignificant differences between the two groups (Table 1). The changes of platelet count during therapy in daily and intermittent eltrombopag dosage groups are shown in Figures 3 and 4, respectively.

\section{Eltrombopag Dosage and Duration}

The median eltrombopag dose was 75 (50-75) $\mathrm{mg}$ daily in the daily eltrombopag dosage group and $50(50-75) \mathrm{mg}$ for 3 (2-3) days/week in the intermittent eltrombopag dosage group. In the intermittent eltrombopag dosage group, 10 out of 11 patients without relapse were controlled on 3 days per week and only one patient was controlled on 2 days/week.

The median duration of eltrombopag therapy and follow-up for daily and intermittent eltrombopag dosage groups were 24 (8-32) weeks and $32(20-32)$ weeks, respectively.

\section{Eltrombopag Effectiveness}

Comparison between daily and intermittent eltrombopag dosage groups as regard CR, PR, and relapse rates showed insignificant differences between the two groups. In the daily eltrombopag dosage group, the OR rate was $9 / 13$ (69.23\%), the CR rate was $7 / 13$ (53.85\%), the PR rate was $2 / 13(15.38 \%)$, and the relapse rate was $4 / 13$ (30.77\%). In the intermittent eltrombopag dosage group, the OR rate was $11 / 16(68.75 \%)$, the $\mathrm{CR}$ rate was $8 / 16(50 \%)$, the PR rate was $3 / 16(18.75 \%)$, and the relapse rate was $5 / 16$ (31.25\%) (Table 1).

Comparison between the two groups as regard discontinuation of eltrombopag due to increase platelet count above $400 \times 10^{9} / \mathrm{L}$ showed significant differences between the two groups. In the daily eltrombopag dosage group, there were four $(30.77 \%)$ patients who discontinue eltrombopag due to an increase in platelet count above $400 \times 10^{9} /$ $\mathrm{L}$, but there was no patient in the intermittent eltrombopag dosage group (Table 1).

In the daily eltrombopag dosage group, there were four (30.77\%) patients who developed bleeding during relapse and needed rescue therapy. In the intermittent eltrombopag dosage group, there were five $(31.25 \%)$ patients who developed bleeding during relapse and needed rescue therapy.

Comparison between daily and intermittent eltrombopag dosage groups as regard relapse-free survival (RFS) showed insignificant differences between the two groups $(P$-value $=0.93)$. 


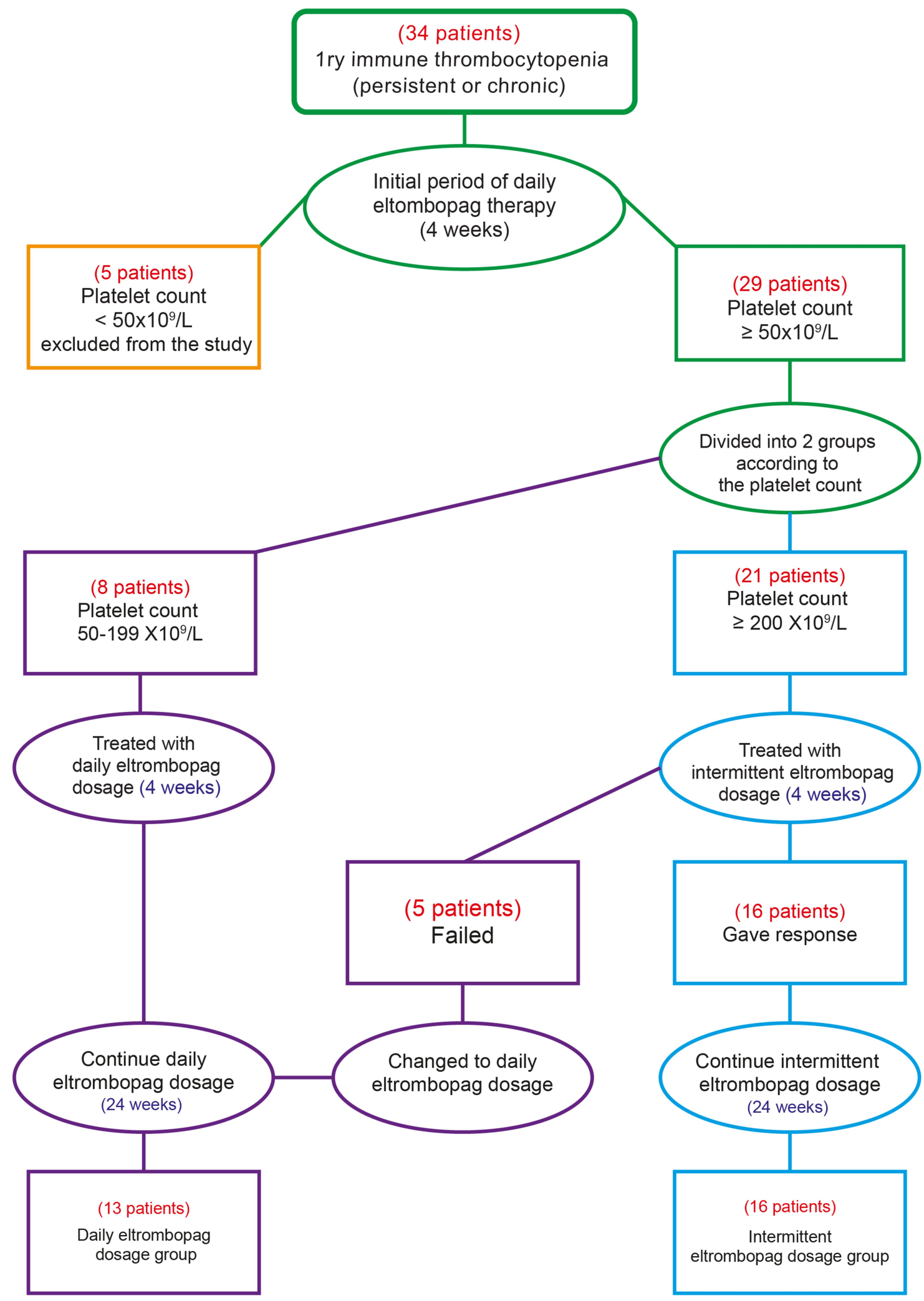

Figure 2 Study design. 


\begin{tabular}{|c|c|c|c|c|c|c|c|c|c|c|c|c|c|c|c|c|c|c|c|c|c|c|}
\hline$\frac{\mathscr{D}}{\frac{\partial}{\pi}}$ & & $\stackrel{\infty}{\widetilde{\infty}} \underset{0}{0}$ & $\stackrel{\widetilde{m}}{0}$ & 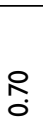 & ô & oे & $\stackrel{m}{0}$ & $\stackrel{\circ}{\circ}$ & $\bar{m}$ & 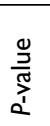 & $\stackrel{\text { ¿ }}{-}$ & 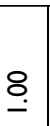 & స్రి & & & $\overline{\bar{o}}$ & & & 虽 & & & 羍 \\
\hline 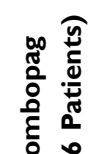 & 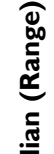 & & & & 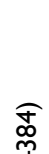 & o & & & & ১゚ & $\underset{\substack{n \\
\infty}}{\stackrel{n}{0}}$ & $\stackrel{\sim}{\sim}$ & $\stackrel{\sim}{\sim}$ & 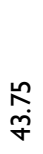 & 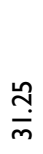 & $\stackrel{\stackrel{\sim}{a}}{m}$ & 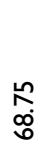 & 0 & 요 & $\begin{array}{l}\stackrel{\tilde{n}}{\underline{\alpha}} \\
\underline{\underline{\alpha}}\end{array}$ & $\stackrel{\stackrel{\leftrightarrow}{\oplus}}{m}$ & 0 \\
\hline 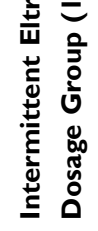 & 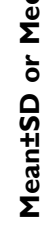 & 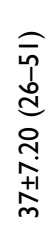 & 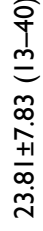 & 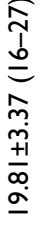 & 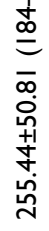 & 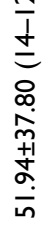 & 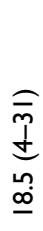 & $\begin{array}{l}\text { స̂ } \\
\text { d } \\
\text { n }\end{array}$ & 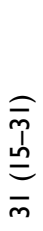 & $\begin{array}{l}\text { ¿ } \\
\text { है } \\
\bar{\xi}\end{array}$ & $=$ & $\sigma$ & $\nabla$ & 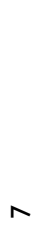 & in & in & $=$ & 0 & $\infty$ & $m$ & in & 0 \\
\hline
\end{tabular}

$$
\text { 品 }
$$

总

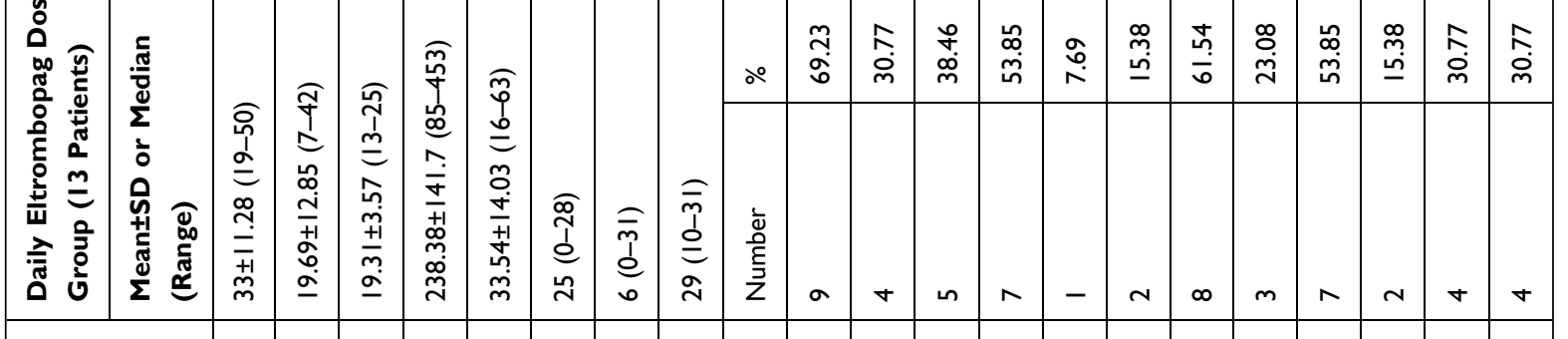

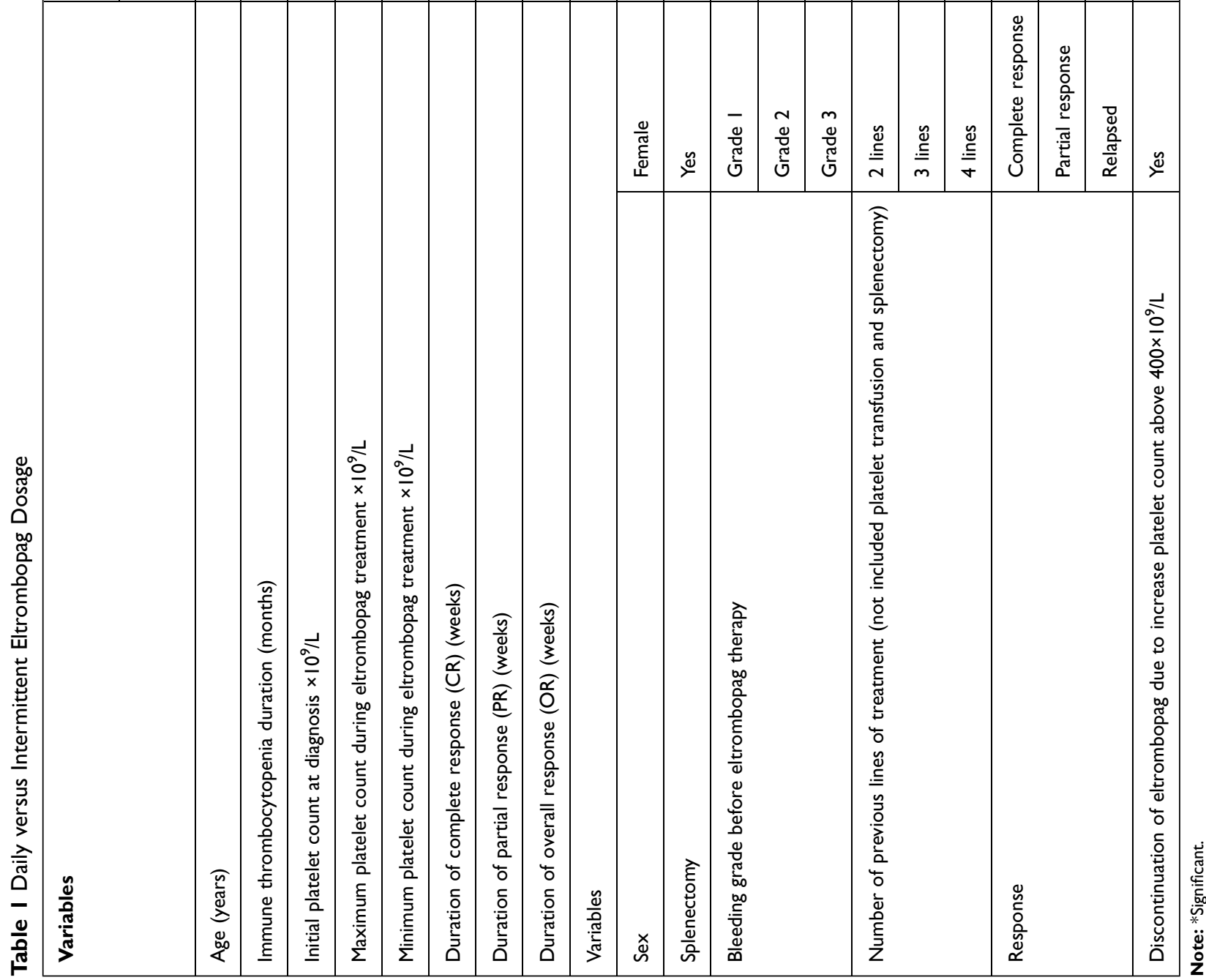




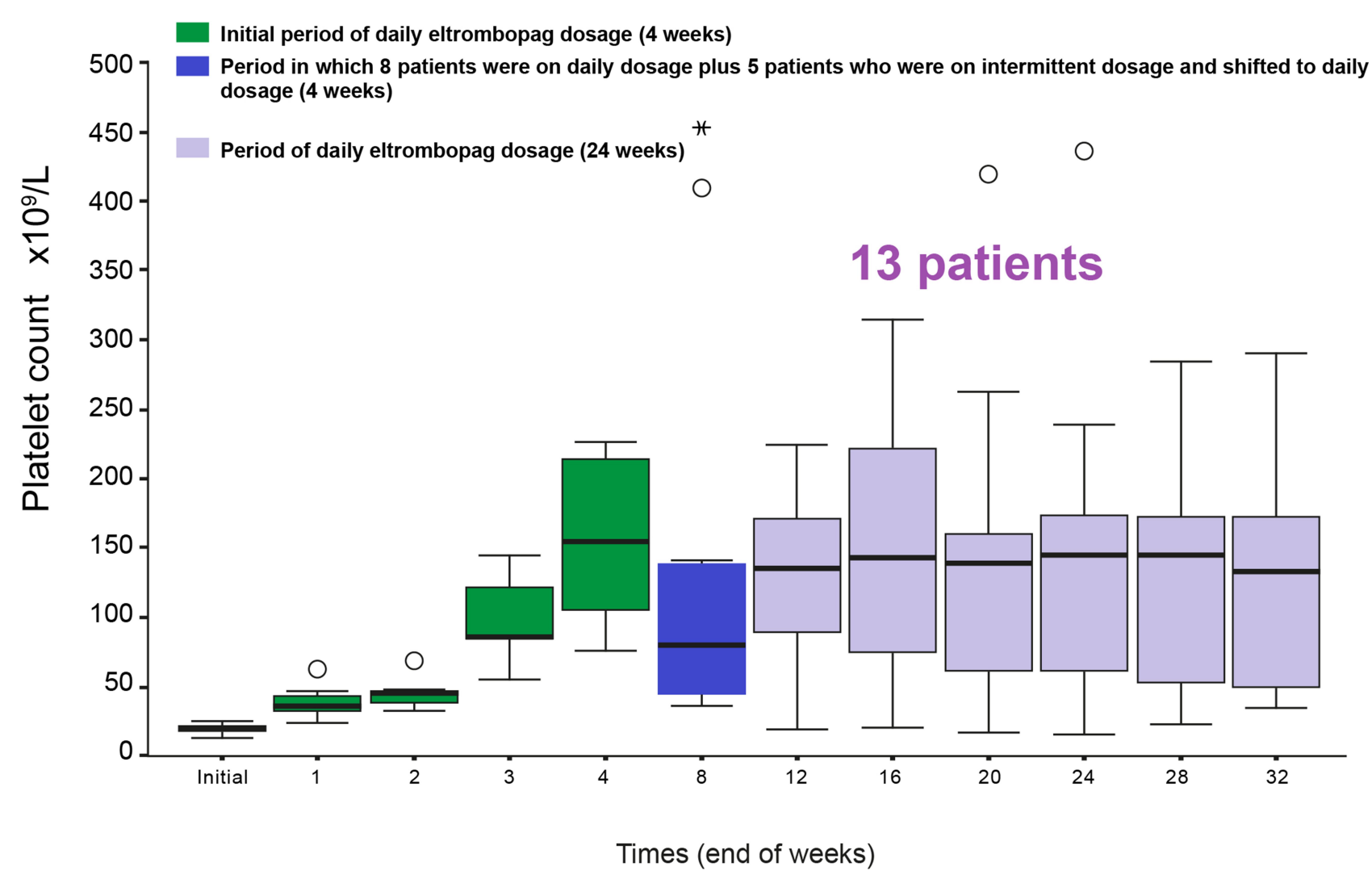

Figure 3 Initial platelet count and changes of platelet count during therapy in daily dosage group.

\section{Eltrombopag Safety}

No serious adverse events were reported. Headache was the most frequently reported adverse event in the daily eltrombopag dosage group $(23.08 \%)$. Headache and fatigue were the most frequently reported adverse events in the intermittent eltrombopag dosage group (12.5\%). Seven (53.85\%) and five (31.25\%) patients developed adverse events in the daily and intermittent eltrombopag dosage groups, respectively. There was insignificance between the two groups as regards development of adverse events (Table 2).

\section{Discussion}

Long-term use of eltrombopag as a maintenance therapy to treat chronic ITP is required in most patients. ${ }^{17,18}$ As it is recommended by the drug's prescribing information, using eltrombopag in chronic ITP necessitates that patients have to follow strict instructions to ensure adequate absorption. The drug should be taken on an empty stomach and at least 4 hours before other medications. Moreover, dietary fat considerably decreases the oral bioavailability of eltrombopag. This may impair patients' quality-of-life and their compliance. ${ }^{19,20}$
The plasma elimination half-life of eltrombopag is 21-32 hours in normal subjects and it is prolonged (26-35 hours) in patients with ITP, and this may make reduction in dose frequency, less than once daily, practical. ${ }^{10,11}$ In addition to eltrombopag half-life in ITP patients, the drug's pharmacokinetic and pharmacodynamic suggest that less-than-daily dosage is reasonable from a biological perspective. ${ }^{21}$

Consequently, we have considered the intermittent eltrombopag dosage protocol for the treatment of chronic ITP, assessing the safety and effectiveness of this protocol on the platelet response and need for rescue therapy in this study. This intermittent eltrombopag dosage may improve patient's compliance as well as be economically more costeffective in many patients, especially in developing countries.

In the present study, the initiation dose of $50 \mathrm{mg}$ once daily was prescribed for all patients as recommended by eltrombopag prescribing information. ${ }^{10}$ However, when dose reduction of the standard daily eltrombopag was indicated for the patients who achieved a platelet counts $\geq 200 \times 10^{9}$, patients were transitioned to the intermittent eltrombopag dosage protocol at the same daily eltrombopag dose, instead of reducing the daily dose. Almost all patients in our study with the 


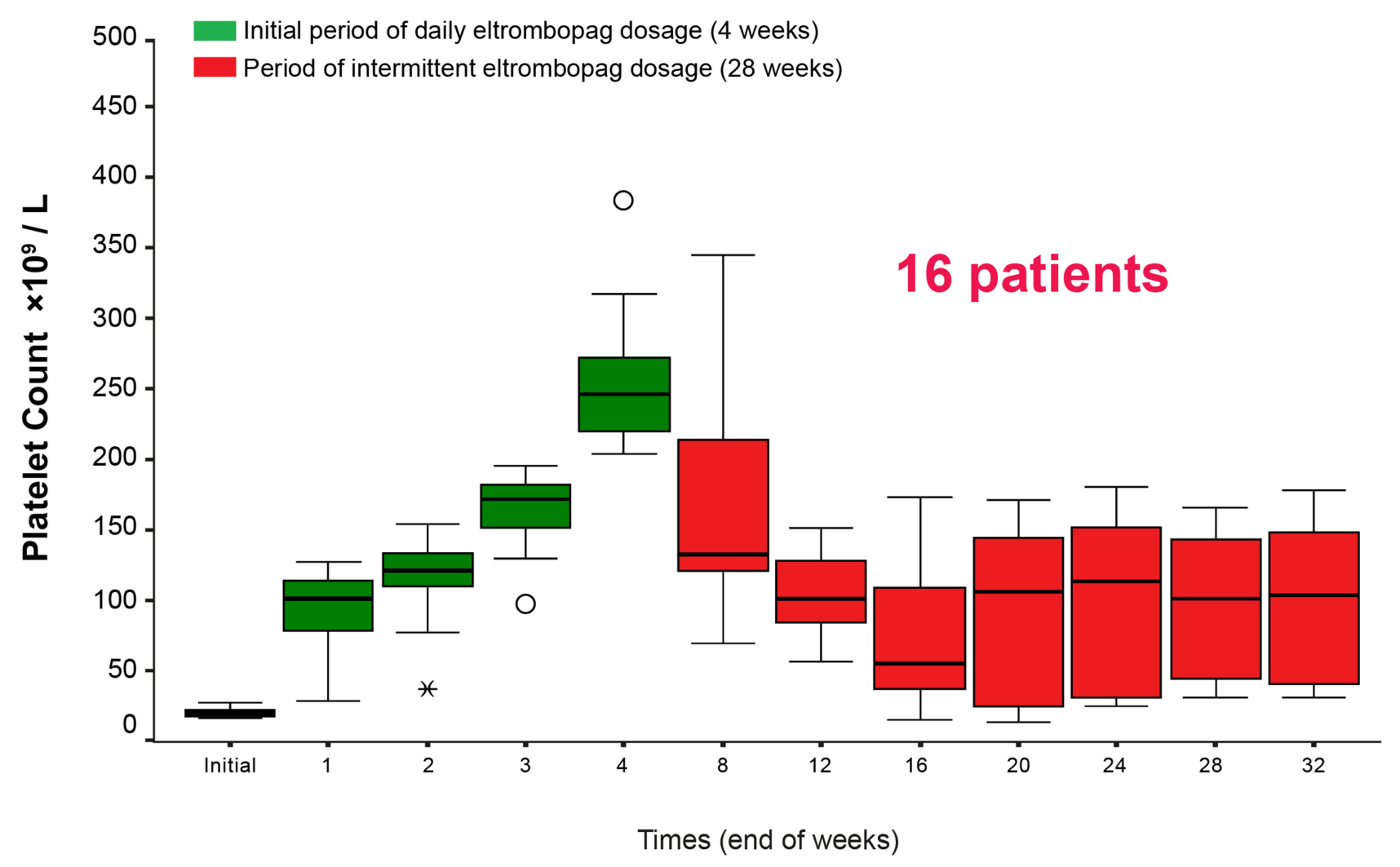

Figure 4 Initial platelet count and changes of platelet count during therapy in intermittent dosage group.

intermittent dosage protocol were controlled 3 days/week dosage and only one patient was controlled on 2 days/week with the median dose of 50 (50-75) $\mathrm{mg}$.

Concerning eltrombopag effectiveness, the result of the present study demonstrated that intermittent eltrombopag dosage was effective in improving platelet counts and its effectiveness was comparable to the standard daily dosage; OR rates were $68.75 \%$ and $69.23 \%$, respectively. The need for rescue therapy in patients with relapse on intermittent eltrombopag dosage was similar to those with the standard daily dosage; $31.25 \%$ and $30.77 \%$, respectively.
These findings were in accordance with that of AlSamkari and Kuter, ${ }^{22}$ who concluded that intermittent eltrombopag dosage was effective in raising the platelet counts and reducing bleeding events for patients with chronic ITP and it was comparable to standard daily dosage. Al-Samkari and $\mathrm{Kuter}^{22}$ found that when receiving intermittent dose eltrombopag, $95 \%, 84 \%$, and $71 \%$ of all platelet counts were $\geq 20 \times 10^{9} / \mathrm{L}, \geq 50 \times 10^{9} / \mathrm{L}$, and $\geq 100 \times 10^{9} / \mathrm{L}$, respectively.

Al-Samkari and $\mathrm{Kuter}^{22}$ documented in their study that $50 \%$ of the patients who received intermittent eltrombopag

Table 2 Incidence of Side-Effects During the Treatment Period in the Studied Patients

\begin{tabular}{|c|c|c|c|c|c|}
\hline \multirow[t]{2}{*}{ Side-Effects } & \multicolumn{2}{|c|}{$\begin{array}{l}\text { Daily Eltrombopag Dosage Group (13 } \\
\text { Patients) }\end{array}$} & \multicolumn{2}{|c|}{$\begin{array}{l}\text { Intermittent Eltrombopag Dosage Group (16 } \\
\text { Patients) }\end{array}$} & \multirow[t]{2}{*}{$P$-value } \\
\hline & Number & $\%$ & Number & $\%$ & \\
\hline Had Side-effects & 7 & 53.85 & 5 & 31.25 & 0.27 \\
\hline Headache & 3 & 23.08 & 2 & 12.5 & 0.63 \\
\hline Fatigue & 2 & 15.38 & 2 & 12.5 & 1.00 \\
\hline Nausea & I & 7.69 & 0 & 0 & 0.45 \\
\hline Diarrhea & I & 7.69 & I & 6.25 & 1.0 \\
\hline
\end{tabular}


dosage required rescue treatment (glucocorticoids or intravenous immunoglobulin) for thrombocytopenia. In those patients, bleeding was limited to WHO grade 1 mucocutaneous bleeding, no patient required transfusion of packed red blood cells, and requirement for rescue treatment was similar to what has been reported in studies examining daily dosage.

Regarding eltrombopag safety, our study showed that it was a safe drug with no apparent serious adverse events. The most frequently observed side-effects were headache, fatigue, nausea, and diarrhea, with no significant difference in patients who were on eltrombopag intermittent dosage and those who were on eltrombopag daily dosage. No patients developed serious adverse events such as thromboembolism, elevated liver enzymes, cataract, serious infection, bone marrow fibrosis, or death during eltrombopag therapy.

These findings were consistent with other studies which suggested that eltrombopag was generally well tolerated. ${ }^{22,23}$

Although a pharmaco-economic analysis was not done in the present study, it is reasonable that this intermittent eltrombopag dosage approach could be cost-effective in comparison to the daily dosage in certain patients. However, such comparison can be investigated as part of other prospective controlled studies to confirm the utility and cost-effectiveness of our approach.

The limitations of the present study included the small number of patients included in this study and the retrospective approach for this study.

\section{Conclusion}

From the previous data in the current study we concluded that intermittent eltrombopag dosage is safe and effective in raising the platelet count in patients with chronic or persistent ITP refractory to prior therapies and comparable to the daily eltrombopag dosage.

The advantages of the use of an alternate dose of eltrombopag in minimizing the adverse events, improving compliance and quality-of-life, and its great cost-effective value for ITP patients need further studies.

\section{Abbreviations}

CR, complete response; F, female; ITP, immune thrombocytopenia; IRB, Institutional Review Board; M, male; NR, no response; OR, overall response; $\mathrm{PR}$, partial response; $\mathrm{R}$, relapse; RFS, relapse-free survival; SD, standard deviation; SPSS, Statistical Package for the Social Sciences; TPO-R, thrombopoietin receptor; WHO, World Health Organization.

\section{Ethics Approval and Consent to Participate}

The study was approved by the research ethical committee of faculty of medicine, Tanta University. Institutional Review Board (IRB) for human studies (Approval code is $34,120 / 9 / 20)$. Our study conforms to provisions of the Declaration of Helsinki. Informed written consent from all patients was taken before the starting of data collection.

\section{Author Contributions}

All authors made substantial contributions to conception and design, acquisition of data, or analysis and interpretation of data; took part in drafting the article or revising it critically for important intellectual content; agreed to submit to the current journal; gave final approval of the version to be published; and agree to be accountable for all aspects of the work.

\section{Disclosure}

The authors declare that they have no competing interests.

\section{References}

1. Lambert MP, Gernsheimer TB. Clinical updates in adult immune thrombocytopenia. Blood. 2017;129(21):2829-2835. doi:10.1182/ blood-2017-03-754119.

2. Audia S, Mahévas M, Samson M, Godeau B, Bonnotte B. Pathogenesis of immune thrombocytopenia. Autoimmun Rev. 2017;16 (6):620-632. doi:10.1016/j.autrev.2017.04.012.

3. Mithoowani S, Gregory-Miller K, Goy J, et al. High-dose dexamethasone compared with prednisone for previously untreated primary immune thrombocytopenia: a systematic review and meta-analysis. Lancet Haematol. 2016;3(10):e489-e496. doi:10.1016/S23523026(16)30109-0.

4. Matschke J, Müller-Beissenhirtz H, Novotny J, et al. A Randomized trial of daily prednisone versus pulsed dexamethasone in treatmentnaïve adult patients with immune thrombocytopenia: EIS 2002 study. Acta Haematol. 2016;136(2):101-107. doi:10.1159/000445420.

5. Cuker A, Cines DB, Neunert CE. Controversies in the treatment of immune thrombocytopenia. Curr Opin Hematol. 2016;23(5):479-485. doi:10.1097/MOH.0000000000000270.

6. Palandri F, Polverelli N, Sollazzo D, et al. Have splenectomy rate and main outcomes of ITPchanged after the introduction of new treatments?A monocentric study in the outpatient setting during 35 years. Am J Hematol. 2016;91(4):E267-E272. doi:10.1002/ajh.24310.

7. Mazza P, Minoia C, Melpignano A, et al. The use of thrombopoietinreceptor agonists (TPO-RAs) in immune thrombocytopenia (ITP): a "real life"retrospective multicenter experience of the ReteEmatologica Pugliese (REP). Ann Hematol. 2016;95(2):239-244. doi:10.1007/s00277-015-2556-z.

8. Elgebaly AS, Ashal GE, Elfil M, Menshawy A. Tolerability and efficacy of eltrombopag in chronic immune thrombocytopenia: meta-analysis of randomized controlled trials. Clin Appl Thromb Hemost. 2017;23 (8):928-937. doi:10.1177/1076029616663849.

9. Brynes RK, Wong RSM, Thein MM, et al. A2-year, longitudinal, prospective study of the effects of eltrombopag on bone marrow inpatients with chronic immune thrombocytopenia. Acta Haematol. 2017;137(2):66-72. doi:10.1159/000452992. 
10. Promacta [Package insert]. Research Triangle Park. NC. GlaxoSmithKline; 2016. Available from: http:/www.accessdata.fda. gov/drugsatfda_docs/label/2016/022291s016,207027s0011bl.pdf. Accessed July 10, 2017.

11. Hayes S, Ouellet D, Zhang J, Wire MB, Gibiansky E. Population PK/ PD modeling of eltrombopag in healthy volunteers and patients with immune thrombocytopenic purpura and optimization of response-guided dosing. J Clin Pharmacol. 2011;51(10):1403-1417. doi:10.1177/0091270010383019.

12. Rodeghiero F, Michel M, Gernsheimer T, et al. Standardization of bleeding assessment in immune thrombocytopenia: report from the International Working Group. Blood. 2013;121(14):2596-2606. doi:10.1182/blood-2012-07-442392.

13. Neunert C, Lim W, Crowther M, Cohen A, Solberg L Jr, Crowther MA. American Society of Hematology. The American Society of Hematology 2011 evidence-based practice guideline for immune thrombocytopenia. Blood. 2011;117(16):4190-4207. doi:10.1182/blood-2010-08-302984.

14. Rodeghiero F, Stasi R, Gernsheimer T, et al. Standardization of terminology, definitions and outcome criteria in immune thrombocytopenic purpura of adults and children: report from an international working group. Blood. 2009;113(11):2386-2393. doi:10.1182/blood2008-07-162503.

15. Fogarty PF, Tarantino MD, Brainsky A, Signorovitch J, Grotzinger KM. Selective validation of the WHO bleeding scale in patients with chronic immune thrombocytopenia. Curr Med Res Opin. 2012;28(1):79-87. doi:10.1185/03007995.2011.644849.

16. Common Terminology Criteria for Adverse Events (CTCAE) Version 5.0; Published November 27, 2017. https://ctep.cancer.gov/protocol development/electronic_applications/docs/CTCAE_v5_Quick_ Reference_8.5x11.pdf. Accessed February 28, 2018.
17. Gonzalez-Lopez TJ, Pascual C, Alvarez-Roman MT, et al. Successful discontinuation of eltrombopag after complete remission in patients with primary immune thrombocytopenia. Am J Hematol. 2015;90(3): E40-43. doi:10.1002/ajh.23900.

18. Ghadaki B, Nazi I, Kelton JG, Arnold DM. Sustained remissions of immune thrombocytopenia associated with the use of thrombopoietin receptor agonists. Transfusion. 2013;53(11):2807-2812. doi:10.1111/ trf. 12139 .

19. Wire MB, Bruce J, Gauvin J, et al. A randomized, open-label, 5-period, balanced crossover study to evaluate the relative bioavailability of eltrombopag powder for oral suspension (PfOS) and tablet formulations and the effect of a high-calcium meal on eltrombopag pharmacokinetics when administered with or 2 hours before or after PfOS. Clin Ther. 2012;34(3):699-709. doi:10.1016/j.clinthera.2012.01.011.

20. Williams DD, Peng B, Bailey CK, et al. Effects of food and antacids on the pharmacokinetics of eltrombopag in healthy adult subjects: two single-dose, open label, randomized-sequence, crossover studies. Clin Ther. 2009;31(4):764-776. doi:10.1016/j.clinthera.2009.04.010.

21. Jenkins JM, Williams D, Deng Y, et al. Phase 1 clinical study of eltrombopag, an oral, non peptide thrombopoietin receptor agonist. Blood. 2007;109(11):4739-4741. doi:10.1182/blood-2006-11-057968.

22. Al-Samkari H, Kuter DJ. An alternative intermittent eltrombopag dosing protocol for the treatment of chronic immune thrombocytopenia. Br J Clin Pharmacol. 2018;84(11):2673-2677. doi:10.1111/bcp.13717.

23. Cheng G, Saleh MN, Marcher C, et al. Eltrombopag for management of chronic immune thrombocytopenia (RAISE): a 6-month, randomised, Phase 3 study. Lancet. 2011;377(9763):393-402. doi:10.1016/ S0140-6736(10)60959-2.
Journal of Blood Medicine

\section{Publish your work in this journal}

The Journal of Blood Medicine is an international, peer-reviewed, open access, online journal publishing laboratory, experimental and clinical aspects of all aspect pertaining to blood based medicine including but not limited to: Transfusion Medicine; Blood collection, Donor issues, Transmittable diseases, and Blood banking logistics; Immunohematology; Artificial and alternative blood based

\section{Dovepress}

therapeutics; Hematology; Biotechnology/nanotechnology of blood related medicine; Legal aspects of blood medicine; Historical perspectives. The manuscript management system is completely online and includes a very quick and fair peer-review system. Visit http://www.dovepress.com/testimonials.php to read real quotes from published authors. 